\title{
Abdominal distention and constipation followed by herpes zoster infection
}

\author{
Eung-Don Kim, Byung-Gil Kang, Jung Hyun Kim, Misun Roh, and Dae Hyun Jo \\ Department of Anesthesiology and Pain Medicine, Daejeon St. Mary's Hospital, College of Medicine, The Catholic University of \\ Korea, Daejeon, Korea
}

Herpes zoster is characterized by a unilateral painful skin rash and bullae according to the dermatome, and is caused by reactivation of the varicella zoster virus (VZV). Peripheral motor neuropathy, called segmental zoster paresis, is an unusual complication of herpes zoster (2.5-9.4\%) [1]. Visceral neuropathy has been also reported, with urinary retention, cystitis, and colonic pseudo-obstruction [2]. Gastrointestinal symptoms caused by herpes zoster infection are extremely rare phenomena. We present a patient who showed the uncommon visceral neuropathy after zoster infection.

A 74-year-old female patient visited our pain clinic due to the painful skin lesions. On exam, the patient had rashes and bullae in the left T-10 dermatome distribution, which was accompanied by allodynia and hyperalgesia. She complained constipation and abdominal distention developed two days after the onset of skin lesion.

Her medical history was unremarkable, and she previously had regular bowel habits and no medication causing constipation. The abdomen was soft, non-tender without distention or palpable mass. Bowel sounds were normal. Laboratory studies were normal, and plain abdominal radiograph had a non specific finding of fecal impaction.

She kept the epidural catheterization and medication with pregabalin $150 \mathrm{mg}$, nortriptyline $10 \mathrm{mg}$ and tramadol/acetaminophen combination tablets $3 \mathrm{~T}$ per day. After relieving the pain, her main complaints were constipation and abdominal distention. Tramadol/acetaminophen combination tablets and nor- triptyline were discontinued and epidural catheter was removed on the seventh day of hospitalization.

For further evaluation of the gastrointestinal symptoms, the patient was transferred to the gastroenterology service at the hospital. The patient underwent colonoscopy, which did not reveal a specific finding besides. The patient was managed with mosapride, trimebutine, magnesium hydroxide, and bowel enemas, and the gastrointestinal symptoms gradually improved over the remainder of the 14 day hospitalization. At follow up clinic visit, the patient reported complete resolution of gastrointestinal and herpes zoster symptoms.

Visceral neuropathy with herpes zoster infection can present with urinary retention, cystitis, and colonic pseudo-obstruction [2]. There is also a report have found severe constipation after lower thoracic segment herpes zoster [3]. In that case, although there was no radiologic finding of bowel obstruction, they postulated that the pathogenesis of intestinal pseudo-obstruction followed by herpes zoster could explain their case.

There were three hypotheses explaining the pathogenesis of herpes zoster induced intestinal pseudo-obstruction. The first is direct viral involvement of the intrinsic intestinal autonomic nervous system through the myenteric plexus. The second is localized parietal and visceral peritoneal inflammation from the vesicular eruption of the overlying dermatome. The third is involvement of extrinsic autonomic nervous system followed by the infection of anterior horn of the spinal cord [3]. Furthermore, Pui et al. [4] have demonstrated evidence of VZV

Corresponding author: Dae Hyun Jo, M.D., Department of Anesthesiology and Pain Medicine, Daejeon St. Mary's Hospital, College of Medicine, The Catholic University of Korea, 520-2, Daeheung-dong, Jung-gu, Daejeon 301-723, Korea. Tel: 82-42-220-9114, Fax: 82-42-242-3500, E-mail: pandjo@naver.com

(c) This is an open-access article distributed under the terms of the Creative Commons Attribution Non-Commercial License (http:// creativecommons.org/licenses/by-nc/3.0/), which permits unrestricted non-commercial use, distribution, and reproduction in any medium, provided the original work is properly cited. 
infection in the muscularis propria and myenteric plexus within resection specimen through the immunohistochemistry study. They postulated that the viral involvement of the neuronal plexus and muscularis propria had led to muscle injury and subsequent intestinal motility disturbance. Generally, the prognosis is good, and conservative medical treatments are adequate in the management of colonic pseudo-obstruction with herpes zoster [3].

In the present case, the patient had complained of constipation and abdominal distention prior to epidural injection, and the symptoms persisted after epidural catheter removal. Although constipation is a known adverse effect of tricyclic antidepressant and pregabalin, the patient's symptom had already begun before administration of medication. Therefore, we could exclude the possibilities for the epidural or medication induced gastrointestinal problem.

Our patient's gastrointestinal symptom, constipation, might be caused by intestinal motility disturbance. Although the abdominal radiograph and colonoscopy did not show a pattern of intestinal obstruction, the hypotheses about pathogenesis of herpes zoster induced intestinal pseudo-obstruction could explain her symptoms.

Except for Ramsay-Hunt syndrome, the rate of herpes zoster infection induced paresis phenomenon has been known to be from 2.5 to $9.4 \%$ [1]. This percentage may increase with electrophysiological studies. The higher percentages of motor involvement is probably a result of electrophysiological diagnosis, which could potentially detected subclinical cases [5].

According to Alshekhlee et al. [5], because $70 \%$ of patients develop herpes zoster in areas where motor involvement is difficult to detect, such as thoracic segments, the low incidence of detectable motor complication may lead clinicians to believe that zoster is a primary sensory neuritis.

Taking these studies into consideration, motor involvement could occur more often than would be expected in subclinical level and the authors supposed a tendency to underestimate motor involvement in actual clinical settings.

Although there was no palpable mass on abdominal wall in this case report, the patient complained of flaccid sensation in the left abdomen. Although performing electromyography (EMG) to abdominal muscles would have been beneficial in the diagnosis, EMG was not performed due to patient's rejection.

In conclusion, gastrointestinal complications from herpes zoster infection are very rare; however, recognition of this complication and its benign nature may help to avoid unnecessary treatment and shorten the period of hospitalization and physicians should have an awareness to possibilities for motor involvement of herpes zoster.

\section{References}

1. Thomas JE, Haward FM Jr. Segmental zoster paresis-a disease profile. Neurology 1972; 22: 459-66.

2. Tribble DR, Church P, Frame JN. Gastrointestinal visceral motor complications of dermatomal herpes zoster: report of two cases and review. Clin Infect Dis 1993; 17: 431-6.

3. Siakir M, Anastasia M, Hakan N, Konstantinos M. Acute constipation due to abdominal herpes zoster: an unusual association. Annals of Gastroenterology 2012; 25: 374-5.

4. Pui JS, Furth EE, Minda J, Montone KT. Demonstration of varicella-zoster virus infection in the muscularis propria and myenteric plexi of the colon in an HIV-positive patient with herpes zoster and small bowel pseudo-obstruction (Ogilvie's syndrome). Am Gastroenterol 2001; 96: 1627-30.

5. Alshekhlee A, Tay E, Buczek M, Shakir ZA, Katirji B. Herpes zoster with motor involvement: discordance between the distribution of skin rash and localization of peripheral nervous system dysfunction. J Clin Neuromuscul Dis 2011; 12: 153-7. 